\title{
Hyperuricemia in Chronic Kidney Disease: a role yet to be explained
}

\author{
Rui Barata, Filipa Cardoso, Tiago Assis Pereira \\ Department of Nephrology, Centro Hospitalar Universitário Lisboa Central, Hospital Curry Cabral, Lisbon, Portugal
}

\section{ABSTRACT}

The role of uric acid as an independent risk factor for chronic kidney disease development and progression is still a matter of discussion. Several observational studies showed a positive association between hyperuricemia and the progression of kidney dysfunction, but others did not, which probably derived from different biases and studies insufficiencies. Moreover, the results from studies on patients in hemodialysis and peritoneal dialysis are even more controversial, with some evidence pointing towards a protective role of uric acid in hemodialysis patients, but not in peritoneal dialysis. In addition to most evidence suggesting a role of uric acid in chronic kidney disease pathogenesis and progression, pharmacological treatment of asymptomatic hyperuricemia is still not indicated, with no consensus on either the uric acid level at which treatment would be beneficial, or the target-level to achieve. There are several studies on the renal benefits of xanthine oxidase inhibitors allopurinol and febuxostat, with heterogeneous results. Most of them showed a renoprotective effect of both drugs, delaying renal disease progression. However, the different results found in other studies makes it difficult to draw definitive conclusions. Despite recent evidence pointing toward an important role of uric acid in the pathogenesis and progression of kidney disorders, and the benefits of its treatment, there are still several unanswered questions, and well-conducted studies are needed to make valid conclusions.

Key-Words: Chronic Kidney Disease, Disease prevention, Urate lowering therapy, Uric acid, Xanthine oxidase inhibitors.

\section{INTRODUCTION}

Hyperuricemia is commonly found in the general population, with a prevalence of about 20 to $25 \%$ in adult men and slightly lower prevalence in pre-menopausal women. In both genders, incidence increases with age ${ }^{1}$. The prevalence of chronic kidney disease (CKD) has been increasing in the past few years, with its morbidity and mortality not only related to the end-stage renal disease (ESRD), but also with cardiovascular disease. As such, every effort must be made to slow its progression.

Emerging evidence suggests that the relationship between hyperuricemia and CKD is more than just a marker of renal dysfunction, playing a much more important role in its pathogenesis and progression, and cardiovascular morbidity-mortality as well. If that association proves to be true, the next question would be when to treat hyperuricemia, and which drugs should be used. Allopurinol has been used for a long time, but febuxostat has been showing growing evidence not only regarding the control of hyperuricemia, but also on slowing CKD progression.

In this review, we will try to answer those questions, while describing the most recent evidence on hyperuricemia as a risk factor for CKD and its management in those patients.

\section{BIOLOGICAL BASES}

\section{Uric Acid Homeostasis}

Uric acid (UA) is a compound endogenously produced by the purine metabolism. These purines, generated in the liver (either from dietary or from endogenous synthesis), are first metabolized into guanine and hypoxanthine, and then into xanthine, which is oxidized into UA by xanthine oxidase ${ }^{2}$. Although the reason is not entirely understood, contrary to other mammals, hominids have lost the enzyme uricase, responsible for converting UA into a more soluble molecule ${ }^{3,4}$. As such, UA is excreted unmetabolized, mainly by the kidney (about twothirds), and about one-third through the intestinal tract ${ }^{5,6}$. Several genes encoding membrane transporters responsible for UA excretion have been identified, such as Abcg2 (ATP-binding cassette subfamily $G$ membrane 2) in the enterocytes and proximal renal tubular epithelium; and others associated with UA reabsorption in the renal tubule, such as URAT1 (urate anion exchanger 1) and GLUT9 (glucose transporter type 9) 7 .

\section{Physiological Functions}

UA has two main functions in the human body. The first is related to its role in the elimination of nitrogenous compounds, such as ammonia and urea. The second is derived from its potent antioxidant activity, which is so important that more than half of normal human's free-radical scavenging capacity in the serum is related to $U A^{8,9}$. This ability is of great importance to the microvascular endothelium, where UA prevents oxidative inactivation of endothelial enzymes, maintaining its ability to mediate vascular dilation in situations of oxidative stress ${ }^{10}$.

Apart from its beneficial biological effects, higher levels of UA have been linked to a higher risk of cardiovascular disease, with several studies describing it as an independent risk predictor of cardiovascular mortality ${ }^{10,11}$. This duality has been named the uric 
acid paradox ${ }^{3}$. A possible explanation, derived primarily from animal studies, is that UA has different actions depending on the surrounding cells and chemical environment ${ }^{12}$. In addition to the extracellular effects related to endothelial cells already described, intracellular UA exerts pro-oxidant effects: it reduces nitric oxide inside endothelial cells, stimulates vascular smooth muscle cells proliferation and promotes production of reactive oxygen species and insulin resistance in adipocytes ${ }^{10}$.

\section{DEFINITION OF HYPERURICEMIA}

The definition of hyperuricemia is still debated, and there is no universal consensus. A practical answer is based on the UA solubility point at physiologic temperature and the $\mathrm{pH}$, which is $6.8 \mathrm{mg} / \mathrm{dl}^{10}$. However, many clinical trials have been using different cut-offs, with levels of UA >7 or $7.5 \mathrm{mg} / \mathrm{dl}$ in men and $>6$ or $6.5 \mathrm{mg} / \mathrm{dl}$ in women ${ }^{5}$. In fact, the UA threshold for pathologic level is still controversial, as the levels in healthy population are very heterogeneous, and a large proportion of individuals with high levels of UA do not develop clinical manifestations of gout ${ }^{10}$

\section{PATHOPHYSIOLOGY OF HYPERURICEMIA IN CKD}

Hyperuricemia has been conceptually determined by two types of causes: overproduction and underexcretion of UA; however, there can be a combination of both mechanisms. Hyperuricemia rises in parallel with glomerular filtration rate (GFR) decline, and is present in about $40 \%$ of patients with CKD stages I to III and $70 \%$ of patients with CKD stage IV or stage $\mathrm{V}^{6}$.

UA may be associated with CKD development and progression through several mechanisms, some of them crystal-independent ${ }^{13}$ : direct toxicity to the kidney; exacerbation of other risk factors for kidney disease, such as hypertension; or by being a marker of the severity of other risk factors, including those attributable to or associated with diabetes and the metabolic syndrome ${ }^{14}$. Some experimental studies suggest that hyperuricemia induces renal injury through renal vasoconstriction mediated by endothelial dysfunction, activation of the renin-angiotensin system, afferent arteriolopathy, and epithelialto-mesenchymal transition in renal tubular cells ${ }^{15}$. Additionally, hyperuricemia increases renin activity, leading to the upregulation of angiotensin II and proliferation of vascular smooth muscle cells ${ }^{15}$. This results in vascular wall thickening, which leads to lumen obliteration and hypoperfusion of the kidney, with tubulointerstitial inflammation and fibrosis, as well as arterial hypertension ${ }^{16}$. Furthermore, preglomerular vasculopathy caused by hyperuricemia disturbs the autoregulatory response of afferent arterioles, prompting glomerular hyperten$\operatorname{sion}^{15}$. Tubulointerstitial fibrosis can also be a result of a UA induced epithelial-to-mesenchymal transition in renal tubular cells ${ }^{17}$. In fact, in animal models, administration of a uricase inhibitor to rats caused tubular dilatation, cortex atrophy and glomerulosclerosis ${ }^{18}$. Recent evidence also confirms an important role of UA induced inflammation, both by the more known crystal-dependent mechanisms, but also by direct effect of soluble urate, through oxidative stress, proinflammatory signaling, autophagy and intracellular immunometabolic sensors $^{19}$.

\section{ASSOCIATIONS BETWEEN HYPERURICEMIA AND CKD}

Despite the vast evidence of an association between higher UA levels and several risk factors for CKD, there are still conflicting results in clinical studies, with several unanswered questions regarding the role of UA as a casual, compensatory or coincidental phenomenon in these patients.

\section{Hyperuricemia CKD: risk factor or risk marker?}

It has long been known that there is a strong association between serum UA (sUA) levels and hypertension. In a meta-analysis regarding this association, Grayson et al showed that hyperuricemia causes a $41 \%$ increase in the risk of developing hypertension, with this association more pronounced in women and a younger population ${ }^{20}$. Although the mechanism for this association is not well understood, it is probably related to UA mediated activation of the renin-angiotensin system, suppression of nitric oxide and direct vascular damage (especially to the renal afferent arterioles) ${ }^{21}$.

UA has long been associated with renal disease. At first, based on studies that showed an increase in the incidence of hyperuricemia in parallel with the decline in GFR, it was considered only a marker of CKD, instead of a risk factor causing $\mathrm{it}^{3}$. However, the association between hyperuricemia and CKD may not be simply a consequence of decrease excretion of UA, and there are several studies confirming the association between hyperuricemia and the development and progression of CKD. In a community-based cohort with 13,388 patients followed for 9 years, baseline sUA levels were associated with increased risk for CKD, with odds ratios (OR) of 1.07 ( $95 \%$ confidence interval - $\mathrm{Cl}$ 1.01-1.14) per each increase of $1 \mathrm{mg} / \mathrm{dl}$ in sUA, in a GFR-based model, even after adjustment for possible confounding parameters ${ }^{14}$. In another prospective study with 21,475 individuals followed for 7 years, patients with slight to moderate elevation of SUA (7.0 to 8.9 $\mathrm{mg} / \mathrm{dl}$ ) were associated with a nearly doubled risk for kidney disease (OR 1.74, with $95 \% \mathrm{Cl} 1.45$ to 2.09 ), and patients with very high sUA $(\geq 9.0 \mathrm{mg} / \mathrm{dl})$ were associated with a threefold risk for kidney disease (OR 3.12, with $95 \%$ confidence interval 2.29 to 4.25$)^{22}$.

However, not every study regarding this issue confirmed the association between UA and incident of CKD and its progression. In a study with 227 patients followed for 7 years, with CKD of non-diabetic etiology, there was no relationship between sUA levels and kidney disease progression when the analysis was adjusted for baseline kidney function parameters ${ }^{23}$. In the MDRD study (Modification of Diet in Renal Disease), with 838 patients with CKD stages 3-4 followed for 10 years, the results showed a positive association of hyperuricemia with all-cause and cardiovascular mortality, but not with kidney failure (HR 1.02, with $95 \% \mathrm{Cl} 0.97$ to 1.07$)^{24}$. Thus, some claim that hyperuricemia is just an epiphenomenon of CKD, but not and independent risk factor ${ }^{25}$.

\section{Hyperuricemia in dialysis patients}

Serum UA levels vary greatly in dialysis patients, whether hemodialysis (HD) or peritoneal dialysis (PD). In HD patients, the mean 
reported level of UA is $7.7 \mathrm{mg} / \mathrm{dL}$ (standard deviation of 1.4$)^{26}$, whereas in PD patients the mean level of UA is $8.5 \mathrm{mg} / \mathrm{dL}$ (standard deviation of 2.1 $)^{27}$. However, these levels are highly variable between studies, and and need careful interpretation, since UA levels in patients receiving dialysis depend on several factors, such as the dialysis prescription or the timing of the sample collection.

In HD, UA clearance is similar to urea ${ }^{28}$, since UA easily diffuses into the dialysis effluent. In an HD session with a standard prescription, the single pooled UA removal ration is $60-70 \%$ with high-flux dialyzers ${ }^{29}$. UA clearance for PD is $10-20 \mathrm{~mL} / \mathrm{min}$, being directly proportional to the dialysate exchange volume and flow rate ${ }^{30}$, and there seems to be no difference between automated and continuous ambulatory $\mathrm{PD}^{31}$.

The relationship between UA and mortality in dialysis patients has been evaluated in several studies, summarized by Murea and Tucker in a recent review ${ }^{10}$, which included 14 studies of patients on HD, and five studies in patients on PD. Regarding patients on HD, nine of the fourteen studies found an inverse association between UA and allcause mortality. In one of those studies ( $n=5827$ ), Latif et al found that higher UA level were associated with lower all-cause mortality, with an hazard ration of 0.95 per $1 \mathrm{mg} / \mathrm{dl}$ higher UA level ${ }^{32}$. However, five of those studies found either no association or a direct association between UA and mortality. As for PD, two of those studies found a direct association between UA and mortality, while the other three studies found none.

There are important differences in the results among those studies, which can be a consequence of several biases. However, there seems to be an important difference between both dialysis techniques, since the inverse association of higher UA levels and lower mortality was specific only for HD. Although there is no clear explanation for these results, there are some hypotheses suggested. Some advocate that higher sUA levels are associated with less mortality because sUA is a marker of better nutrition. However, some studies showed that, even after adjustment for nutritional parameters, there was still an association between sUA and mortality. In one of those studies ${ }^{33}$, a prospective study with 261 patients on HD, the authors demonstrated an association between higher levels of sUA and a lower hazard ratio for hospitalization and cardiovascular and all-cause death, even after adjustment for nutritional parameters. Thus, despite the fact that hyperuricemia is related to better nutritional status, this does not seem to be the explanation for the lower mortality in HD patients. Yet, this argument cannot explain the difference found between HD and PD patients.

Another explanation for these results relies on the timing of measurement of sUA levels in HD patients. Given that UA is dialysated in a similar way to urea, levels will fluctuate greatly between HD sessions, and the pre-HD concentration does not reflect the mean levels in those patients. Some studies show that after a HD session, sUA levels tend to decrease by $64.6 \%{ }^{34}$, which means that, between two sessions, those patients will have sUA between those levels. Thus, a patient with normal or low sUA before HD would have a much lower weekly time-averaged mean sUA, with levels probably lower than the necessary concentration for UA to execute its normal antioxidant functions. Likewise, patients with high sUA levels before HD would have near-normal mean sUA levels weekly time-averaged ${ }^{10}$. Although this is an unconfirmed hypothesis, it could explain the protective effects of higher pre-HD sUA levels, and the difference between HD and PD patients.

\section{Hyperuricemia in transplant patients}

Hyperuricemia is frequent in renal transplant patients, normally occurring early in the post-transplant period ${ }^{25}$. Frequent risk factors for hyperuricemia in these patients include the use of calcineurin inhibitors (cyclosporine being the most common risk factor for hyperuricemia in the post-transplant period ${ }^{35}$ ) or diuretics, reduced glomerular filtration rate (GFR), obesity, recipient age and pre-existing history of hyperuricemia ${ }^{36}$.

However, the evidence regarding the effects of hyperuricemia on renal allograft function and survival is very heterogeneous and inconclusive ${ }^{37}$. Some studies showed an inverse association between hyperuricemia and renal allograft function and survival ${ }^{38-40}$, suggesting a role for hyperuricemia as an independent risk factor. However, this association is not evident in other studies, which conclude that SUA is merely a marker of graft dysfunction ${ }^{41-43}$.

The evidence regarding treatment of asymptomatic hyperuricemia is also controversial. However, lifestyle measures and avoidance of cyclosporine and diuretics are acceptable strategies to reduce sUA levels ${ }^{36}$.

\section{TREATMENT OF HYPERURICEMIA IN CKD}

\section{General principles}

As a result of the lack of strong evidence regarding the role of hyperuricemia in patients without gout or nephrolithiasis, current guidelines do not support the treatment of isolated hyperuricemia with urate lowering therapy. However, advice on lifestyle and the treatment of associated comorbidities is always advised ${ }^{44}$.

Despite the limited effect of lifestyle modification on sUA and the lack of evidence from RCTs, every patient with hyperuricemia should receive appropriate advice on lifestyle recommendations to lower sUA. Furthermore, most of these measures are useful in the vast majority of the comorbidities that patients with hyperuricemia frequently have ${ }^{45}$. However, given their limited effect, they should be considered an adjunctive, and should not replace pharmacologic treatment, whenever indicated ${ }^{46}$. Those measures include:

- Reduce weight and daily exercise, even though the effects are only modest (losing $7.7 \mathrm{~kg}$ decreases, in average, $1.6 \mathrm{mg} / \mathrm{dL}$ in $\mathrm{s} \cup \mathrm{A})^{47}$

- Decrease consumption of purine-rich meat and seafood;

- Reduce energy drinks and soft drinks sweetened with fructose corn syrup 45 ;

- Limit the intake of beer, wine and spirits ${ }^{46}$;

- Minimize the use of drugs that increase sUA, such as diuretics, beta-blockers or low dose of acetylsalicylic acid ${ }^{3}$. 


\section{Urate-lowering agents}

There are 3 main classes of urate-lowering agents: uricosuric agents, which increase kidney UA excretion; xanthine oxidase inhibitors (allopurinol and febuxostat), which block the conversion of purine metabolites to UA; and recombinant uricases, which oxidize UA into a highly soluble product ${ }^{48}$.

\section{Allopurinol}

Allopurinol is a xanthine oxidase inhibitor, and it is metabolized into oxypurinol, which is excreted by the kidneys. For this reason, and since allopurinol may cause hypersensitive reactions, its dose must be reduced in patients with CKD. In patients with an eGFR $<60 \mathrm{~mL} /$ $\mathrm{min} / 1.73 \mathrm{~m}^{2}$, it is recommended to start at a dose of $50-100 \mathrm{mg} /$ day, increasing the dose to 200 or $300 \mathrm{mg} /$ day after 2-5 weeks to achieve serum UA levels of $<6 \mathrm{mg} / \mathrm{dL}$. It is acceptable to use doses $>300 \mathrm{mg} /$ day, as long as there is proper monitoring of toxicity ${ }^{48}$. Its main adverse events are related to allopurinol hypersensitivity syndrome, characterized by skin rash, eosinophilia, hepatitis and fever, a rare but potentially life-threatening condition ${ }^{49}$.

There is generally a scarcity of RCT regarding effects of control of hyperuricemia in CKD patients. In a 2013 systematic review of 8 RCT, Bose et al compared treatment of hyperuricemia with allopurinol versus placebo and its effect on the retardation of $C K D^{50}$. In 5 of these studies, they found no significant differences in eGFR among patients in each group. The other 3 trials showed a slight benefit on the allopurinol arm, but no significant changes in proteinuria or blood pressure. However, there was an important heterogeneity in the monitorization of kidney function and the follow-up among these studies.

In another meta-analysis which included 19 RCTs, Kanji et al found significant benefits with allopurinol therapy in terms of reducing serum UA levels, blood pressure control and a slight improvement in eGFR ${ }^{51}$.

\section{Febuxostat}

Febuxostat is a newer xanthine oxidase inhibitor, with more efficacy and specificity than allopurinol for xanthine oxidase $\mathrm{s}^{52}$. Its elimination is mainly by the liver and bile, and it is less associated with hypersensitivity than allopurino ${ }^{53}$. Its main adverse effects include liver function abnormalities, diarrhea and rash. It should not be used simultaneously with azathioprine or mercaptopurine, since those drugs are metabolized by xanthine oxidase and their levels may increase, causing toxic$i{ }^{54}$. Regarding its safety in renal disease, it can be used without dose reduction in patients with eGFR $\geq 30 \mathrm{~mL} / \mathrm{min}$. However, most of the safety studies included only a few patients with CKD stages $\geq 4$, and the studies in patients on dialysis are even fewer, which is why the FDA has not yet approved febuxostat in that population. Other than that, it appears to be well tolerated and efficient. Thus, it should be used with dose reduction in patients with CKD stages 4-546,55,56.

In spite of being a newer drug than allopurinol, there are already several published studies demonstrating its beneficial effects on renal function. In a post-hoc analysis of the FOCUS (Febuxostat Open-label
Clinical trial of Urate-lowering efficacy and Safety) study, with 116 hyperuricemic gout patients receiving febuxostat at different doses $(40,80$, or $120 \mathrm{mg}$ ) for 5 years, there was a clear benefit on renal function, with maintenance (or even improvement) in eGFR that was inversely correlated with the amount of reduction in $\mathrm{SUA}$, with an expected improvement in eGFR of $1 \mathrm{~mL} / \mathrm{min}$ for every $1 \mathrm{mg} / \mathrm{dL}$ reduction in $\mathrm{SUA}^{57}$.

In the EXCEL (Febuxostat/Allopurinol Comparative Extension LongTerm) study $(n=1086)$, patients were treated with either febuxostat ( 80 or $120 \mathrm{mg}$ daily) or allopurinol (300 mg daily). The febuxostat group showed significant benefits of renal function, with greater reductions in sUA associated with less decline in renal function (preservation of $1.2 \mathrm{~mL} / \mathrm{min} / 1.73 \mathrm{~m}^{2}$ for every $1 \mathrm{mg} / \mathrm{dL}$ reduction in $\left.\mathrm{s} U A\right)^{58}$.

However, some studies failed to show beneficial renal outcomes with febuxostat. More recently, a randomized trial with 467 patients with stage 3 CKD, receiving either placebo of febuxostat, showed no significant difference in renal function within the febuxostat, with similar mean eGFR slopes between both groups ${ }^{59}$.

There are several ongoing trials regarding the effects of uratelowering therapy with febuxostat, as conclusive evidence is needed.

\section{Other pharmacological agents}

Uricosuric agents include benzbromarone, lesinurad, probenecid and sulfinpyrazone. Despite being effective in reducing SUA in patients with normal kidney function, their use is limited in CKD patients, being contraindicated for stage IV CKD (lesinurad is contraindicated if creatinine clearance $<45 \mathrm{~mL} / \mathrm{min}$ ), including dialysis patients ${ }^{46}$. The evidence regarding their benefit on slowing renal dysfunction is scarce, but there are some studies running regarding this issue ${ }^{45}$. Also, their use must be avoided in patients with past history of nephrolithiasis, and are contraindicated in patients with uricosuria greater that 700 to $800 \mathrm{mg} / 24 \mathrm{~h}^{46}$. Further, there are some issues concerning their safety, with lesinurad being associated with acute kidney injury when used alone (which is the reason it needs to be co-prescribed with a xanthine oxidase inhibitor), and benzbromarone being associated with hepatotoxicity 60 .

Other drugs commonly used for other medical indications but with uricosuric properties include losartan, fenofibrate, SGLT2 inhibitors, vitamin $C$ and high doses of acetylsalicylic acid ${ }^{45,46}$.

Pegloticase, a recombinant uricase, is approved for use in patients with gout that is refractory to oral agents for hyperuricemia. It is administered intravenously, and can be used in patients with advanced CKD and dialysis patients ${ }^{46}$. Rasburicase is another recombinant uricase which is approved for use in patients with malignancies receiving chemotherapy, when tumor lysis syndrome is expected ${ }^{61}$.

\section{CONCLUSION}

The association between hyperuricemia and CKD has long been known. However, although many observational studies have identified associations between hyperuricemia and the onset and progression 
CKD, some have not. Thus, although much evidence points toward a role of SUA in the pathogenesis of CKD, the current evidence cannot conclude for certain that it is not just a marker of kidney dysfunction.

Regarding treatment of hyperuricemia, and as a consequence of the heterogeneous evidence on the role of UA in CKD, there is still no indication for treating asymptomatic patients. Although there are several observational studies evaluating the effect of urate lowering therapy on the development and progression of CKD, the results are heterogeneous, much derived from several biases, including the small number of patients included in much of them, and the short followup. For more clear and accurate results, large randomized controlled trials are needed. Besides that, there are still some doubts on whether the renoprotective effects of allopurinol and febuxostat found in some studies are related to their effect on SUA levels, or to their xanthine oxidase inhibition. Another question yet to be answered is the sUA level at which pharmacological therapeutic must be started, and the optimal target level to slow CKD progression.

Concluding, although there are already some insights into the role of hyperuricemia in CKD, and the potential renoprotective effects of urate lowering therapy, the current evidence is still not strong enough to make valid conclusions, and well-conducted studies are needed.

\section{Disclosure of potential conflicts of interest: none declared}

\section{References}

1. Roddy E, Choi HK. Epidemiology of gout. Rheum Dis Clin North Am. 2014;40(2):155-1575.

2. Edwards NL. the Role of hyperuricemia and gout. Clevel Clin J Med Vol. 2008;13-16.

3. Borghi C, Rosei EA, Bardin T, Dawson J, Dominiczak A, Kielstein JT, et al. Serum uric acid and the risk of cardiovascular and renal disease. J Hypertens. 2015;33(9):1729-1741.

4. Wu X, Muzny DM, Chi Lee C, Thomas Caskey C. Two independent mutational events in the loss of urate oxidase during hominoid evolution. J Mol Evol. 1992;34(1):78-84.

5. Mallat SG, Al Kattar S, Tanios BY, Jurjus A. Hyperuricemia, hypertension, and chronic kidney disease: an emerging association. Curr Hypertens Rep. 2016;18(10)

6. Sah OSP, Qing YX. Associations between hyperuricemia and chronic kidney disease: a review. Nephrourol Mon. 2015;7(3).

7. Maiuolo J, Oppedisano F, Gratteri S, Muscoli C, Mollace V. Regulation of uric acid metabolism and excretion. Int J Cardiol. 2016;213:8-14.

8. Becker BF. Towards the physiological function of uric acid. Free Radic Biol Med. 1993;14(6):615-631.

9. El Ridi R, Tallima H. Physiological functions and pathogenic potential of uric acid: a review. J Adv Res. 2017;8(5):487-493.

10. Murea M, Tucker BM. The physiology of uric acid and the impact of end-stage kidney disease and dialysis. Semin Dial. 2019;32(1):47-57.

11. Sánchez-Lozada LG, Lanaspa MA, Cristóbal-García M, García-Arroyo F, Soto V, Cruz-Robles D, et al. Uric acid-induced endothelial dysfunction is associated with mitochondrial alterations and decreased intracellular ATP concentrations. Nephron - Exp Nephrol. 2013;121(3-4):71-78.

12. Johnson RJ, Nakagawa T, Jalal D, Sánchez-Lozada LG, Kang DH, Ritz E. Uric acid and chronic kidney disease: which is chasing which? Nephrol Dial Transplant. 2013;28(9):2221-2228.

13. Jalal DI, Chonchol M, Chen W, Targher G. Uric acid as a target of therapy in CKD. Am J Kidney Dis. 2013;61(1):134-146

14. Weiner DE, Tighiouart H, Elsayed EF, Griffith JL, Salem DN, Levey AS. Uric acid and incident kidney disease in the community. 2008;1204-1211.

15. Uchida S, Kumagai T, Chang WX, Tamura Y, Shibata S. Time to target uric acid to retard chronic kidney disease progression. Contrib Nephrol. 2018;192:56-68.

16. Jalal DI, Maahs DM, Hovind P, Nakagawa T. Uric acid as a mediator of diabetic nephropathy. Semin Nephrol. 2011;31(5):459-465.

17. Kang DH. Hyperuricemia and progression of chronic kidney disease: role of phenotype transition of renal tubular and endothelial cells. Contrib Nephrol. 2018;192:48-55.

18. Sánchez-Lozada LG, Tapia E, Avila-Casado C, Soto V, Franco M, Santamaría J, et al. Mild hyperuricemia induces glomerular hypertension in normal rats. Am J Physiol - Ren Physiol. 2002;283(5 52-5):1105-1110.

19. Joosten LAB, Crişan TO, Bjornstad P, Johnson RJ. Asymptomatic hyperuricaemia: a silent activator of the innate immune system. Nat Rev Rheumatol. 2019; INCOMPLETE

20. Grayson PC, Young Kim S, Lavalley M, Choi HK. Hyperuricemia and incident hypertension: a systematic review and meta-analysis. Arthritis Care Res 2011;63(1):102-110.
21. Viazzi F, Leoncini G, Ratto E, Pontremoli R. Hyperuricemia and renal risk. High Blood Press Cardiovasc Prev. 2014;21(3):189-194

22. Obermayr RP, Temml C, Gutjahr G, Knechtelsdorfer M, Oberbauer R, Klauser-Braun R. Elevated uric acid increases the risk for kidney disease. J Am Soc Nephrol. 2008;19(12):2407-2413.

23. Sturm G, Kollerits B, Neyer U, Ritz E, Kronenberg F. Uric acid as a risk factor for progression of non-diabetic chronic kidney disease? The Mild to Moderate Kidney Disease (MMKD) Study. Exp Gerontol. 2008;43(4):347-352.

24. Madero M, Sarnak MJ, Wang X, Greene T, Beck GJ, Kusek JW, et al. Uric acid and long-term outcomes in CKD. Am J Kidney Dis. 2009;53(5):796-803.

25. Dousdampanis P, Trigka K, Musso CG, Fourtounas C. Hyperuricemia and chronic kidney disease: an enigma yet to be solved. Ren Fail. 2014;36(9):1351-1359.

26. Hsu SP, Pai MF, Peng Y Sen, Chiang CK, Ho TI, Hung KY. Serum uric acid levels show a "J-shaped" association with all-cause mortality in haemodialysis patients. Nephrol Dial Transplant. 2004;19(2):457-462.

27. Feng S, Jiang L, Shi Y, Shen $H$, Shi X, Jin D, et al. Uric acid levels and all-cause mortality in peritoneal dialysis patients. Kidney Blood Press Res. 2013;37(2-3):181-189.

28. Müller-Deile J, Lichtinghagen R, Haller H, Schmitt R. Online Kt/V monitoring in haemodialysis by UV absorbance: variations during intra-dialytic meals. Blood Purif. 2014;37(2):113-118.

29. Kadkhodaee M, Hemmati M, Zahmatkesh M, Ghaznavi R, Mirershadi F, Mahdavi-Mazde M, et al. Assessment of plasma antioxidant status in hemodialysis patients. Ther Apher Dial. 2008;12(2):147-151.

30. Robson M, Oreopoulos DG, Izatt S, Ogilvie R, Rapoport A, deVeber GA. Influence of exchange volume and dialysate flow rate on solute clearance in peritoneal dialysis. Kidney Int. 1978;14(5):486-490.

31. Eloot S, Vanholder R, Dequidt C, Van Biesen W. Removal of different classes of uremic toxins in APD vs CAPD: a randomized cross-over study. Perit Dial Int. 2015;35(4):436-442.

32. Latif W, Karaboyas A, Tong L, Winchester JF, Arrington CJ, Pisoni RL, et al. Uric acid levels and all-cause and cardiovascular mortality in the hemodialysis population. Clin J Am Soc Nephrol. 2011;6(10):2470-2477.

33. Beberashvili I, Sinuani I, Azar A, Shapiro G, Feldman L, Stav K, et al. Serum uric acid as a clinically useful nutritional marker and predictor of outcome in maintenance hemodialysis patients. Nutrition. 2015;31(1):138-147.

34. Shahbazian H, Moghadam AZ, Ehsanpour A, Khazaali M. Changes in plasma concentrations of hypoxanthine and uric acid before and after hemodialysis. Iran J Kidney Dis. 2009;3(3):151-155.

35. Einollahi B, Einollahi $\mathrm{H}, \mathrm{Nafar} \mathrm{M}$, Rostami Z. Prevalence and risk factors of hyperuricemia among kidney transplant recipients. Indian J Nephrol. 2013;23(3):201-205.

36. Mazali FC, Mazzali M. Uric acid and transplantation. Semin Nephrol. 2011;31(5):466-471.

37. Bellomo G. Asymptomatic hyperuricemia following renal transplantation. World J Nephrol. 2015;4(3):324.

38. Akalin E, Ganeshan SV, Winston J, Muntner P. Hyperuricemia is associated with the development of the composite outcomes of new cardiovascular events and chronic allograft nephropathy. Transplantation. 2008;86(5):652-658.

39. Min S II, Yun IJ, Kang JM, Park YJ, Min SK, Ahn C, et al. Moderate-to-severe early-onset hyperuricaemia: a prognostic marker of long-term kidney transplant outcome. Nephrol Dial Transplant. 2009;24(8):2584-2590.

40. Weng SC, Shu KH, Tarng DC, Cheng CH, Chen $\mathrm{CH}$, Yu TM, et al. Uric acid is highly associated with kidney allograft survival in a time-varying analysis. Transplant Proc. 2014;46(2):505-510.

41. Akgul A, Bilgic A, Ibis A, Ozdemir FN, Arat Z, Haberal M. Is uric acid a predictive factor for graft dysfunction in renal transplant recipients? Transplant Proc. 2007;39(4):1023-1026.

42. Meier-Kriesche HU, Schold JD, Vanrenterghem Y, Halloran PF, Ekberg H. Uric acid levels have no significant effect on renal function in adult renal transplant recipients: evidence from the symphony study. Clin J Am Soc Nephrol. 2009;4(10):1655-1660.

43. Boratyńska M, Karbowska A, Klinger M. The effect of hyperuricemia on endothelial biomarkers and renal function in kidney allograft recipients. Transplant Proc. 2010;42(10):4074-4077.

44. Zhang W, Doherty M, Bardin T, Pascual E, Barskova V, Conaghan P, et al. EULAR evidence based recommendations for gout. Part II: Management. Report of a task force of the EULAR Standing Committee for International Clinical Studies Including Therapeutics (ESCISIT). Ann Rheum Dis. 2006;65(10):1312-1324

45. Ramirez-Sandoval JC, Madero M. Treatment of hyperuricemia in chronic kidney disease. Contrib Nephrol. 2018;192:135-146.

46. Vargas-Santos AB, Neogi T. Management of gout and hyperuricemia in CKD. Am J Kidney Dis. 2017;70(3):422-439.

47. Dessein PH, Shipton EA, Stanwix AE, Joffe BI, Ramokgadi J. Beneficial effects of weight loss associated with moderate calorie/carbohydrate restriction, and increased proportional intake of protein and unsaturated fat on serum urate and lipoprotein levels in gout: a pilot study. Ann Rheum Dis. 2000;59(7):539-543.

48. Khanna D, Fitzgerald JD, Khanna PP, Bae S, Singh MK, Neogi T, et al. 2012 American college of rheumatology guidelines for management of gout. part 1: systematic nonpharmacologic and pharmacologic therapeutic approaches to hyperuricemia. Arthritis Care Res. 2012;64(10):1431-1446.

49. Hande KR, Noone RM, Stone WJ. Severe allopurinol toxicity. Am J Med. 1984;76(1):47-56.

50. Bose B, Badve SV, Hiremath SS, Boudville N, Brown FG, Cass A, et al. Effects of uric acid-lowering therapy on renal outcomes: a systematic review and meta-analysis. Nephrol Dial Transplant. 2014;29(2):406-413.

51. Kanji T, Gandhi M, Clase CM, Yang R. Urate lowering therapy to improve renal outcomes in patients with chronic kidney disease: systematic review and meta-analysis. BMC Nephrol. 2015;16(1).

52. Takano Y, Hase-Aoki K, Horiuchi H, Zhao L, Kasahara Y, Kondo S, et al. Selectivity of febuxostat, a novel non-purine inhibitor of xanthine oxidase/xanthine dehydrogenase. Life Sci. 2005;76(16):18351847.

53. Grabowski BA, Khosravan R, Vernillet L, Mulford DJ. Metabolism and excretion of [14C] febuxostat, a novel nonpurine selective inhibitor of xanthine oxidase, in healthy male subjects. J Clin Pharmacol. 2011;51(2):189-201. 
54. Kathleen J, Orcid L. Accepted article. J Subst Abuse Treat. 1996;13(3):287-288.

55. Lim DH, Oh JS, Ahn SM, Hong S, Kim YG, Lee CK, et al. Febuxostat in hyperuricemic patients with advanced CKD. Am J Kidney Dis. 2016;68(5):819-821.

56. Horikoshi R, Akimoto T, Inoue M, Morishita Y, Kusano E. Febuxostat for hyperuricemia: experience with patients on chronic hemodialysis treatment. Clin Exp Nephrol. 2013;17(1):149-150.

57. Whelton A, MacDonald PA, Zhao L, Hunt B, Gunawardhana L. Renal function in gout: long-term treatment effects of febuxostat. J Clin Rheumatol. 2011;17(1):7-13.

58. Whelton A, MacDonald PA, Chefo S, Gunawardhana L. Preservation of renal function during gout treatment with febuxostat: a quantitative study. Postgrad Med. 2013;125(1):106-114.

59. Kimura K, Hosoya T, Uchida S, Inaba M, Makino H, Maruyama S, et al. febuxostat therapy for patients with stage 3 CKD and asymptomatic hyperuricemia: a randomized trial. Am J Kidney Dis. 2018;72(6):798-810.
60. Kydd ASR, Seth R, Buchbinder R, Edwards CJ, Bombardier C. Uricosuric medications for chronic gout. Cochrane Database Syst Rev. 2014;2014(11).

61. Strilchuk L, Fogacci F, Cicero AF. Safety and tolerability of available urate-lowering drugs: a critical review. Expert Opin Drug Saf. 2019;18(4):261-271.

\section{Correspondence to:}

Rui Barata, MD.

Department of Nephrology, Centro Hospitalar Universitário Lisboa Central Rua da Beneficência 8, 1069-166 Lisbon, Portugal.

E-mail: rui.f.barata@gmail.com 